\title{
Maîtrise des impacts environnementaux par la modélisation du système physique
}

\author{
Leila Boubaker ${ }^{(1)}$, Mébarek Djebabra ${ }^{(1)}$ Natacha Gondran ${ }^{(2)}$ \& Hecène Chaabane ${ }^{(3)}$ \\ ( I) Laboratoire LRPI - Institut d'Hygiène et Sécurité Industrielle - Université de Batna, Algérie. \\ (2) Centre SITE de l'École des mines de Saint-Étienne, France \\ (3) Laboratoire de Productique - Université de Batna, Algérie \\ Pour toute correspondance : boubaker.leila@yahoo.fr
}

\section{Résumé}

La certification ISO |400 | connait une diffusion significative. En effet, sous les effets conjugués de la dégradation des milieux naturels et de la concurrence, l'entreprise d'aujourd'hui doit adopter une stratégie environnementale au niveau de toutes ses activités, par la mise en place d'un Système de Management Environnemental (SME).

L'environnement est au c?ur des systèmes de production obligeant ainsi les entreprises à s'interroger et à rendre compte de leurs performances environnementales. Ainsi, une analyse de toutes les activités de l'entreprise fait ressortir des Impacts Environnementaux Significatifs (AES). Afin de faire une première étape vers l'intégration de l'environnement, ces Aspects doivent être maîtrisés par des actions correctives au niveau des installations. Pour ce faire, il faut modéliser le système opérationnel d'où l'utilisation de la méthode GRAICO, laquelle serra compléter par la méthode Analyse des Défaillances et leurs Conséquences (ADC) pour maitriser ces impacts.

Mots-clé : ISO |400I, système de production, modélisation, GRAICO, activités, analyse, impacts environnementaux significatifs.

\begin{abstract}
The certification ISO |400I knows a significant distribution (broadcasting). Indeed, under the combined effects of the degradation of the natural circles and the competition, the current company has to adopt an environmental strategy at the level of all its activities, by the implementation of an Environmental Management system (EMS).

The environment is in the heart of the systems of production so obliging companies to wonder and to report their environmental performances. So, an analysis of all the activities of the company highlights Significant Environmental Impacts (SEI). To make a first stage towards the integration of the environment, these SEl must be mastered by corrective actions at the level of the installations. To do it, it is necessary to model the operational system where from the use of the method GRAICO, which squeezed (tightened) to complete by the method Analyzes Failures and their Consequences (ADC) for controlled these impacts.
\end{abstract}

Keywords : ISO |400I, system of production, modeling, GRAICO, activities, analysis, significant environmental impacts.

\section{Introduction}

Dans le monde d'une économie de marché, les entreprises industrielles vivent une ère caractérisée par un changement dont la conséquence est l'addition de plusieurs facteurs : des marchés de plus en plus incertains, évolution des exigences des clients, du public et de la réglementation. Comme tout autre système les entreprises sont contraintes d'évoluer sous la pression de leur environnement (Mirdamadi, 2009). Pour anticiper ces contraintes et pouvoir s'adapter face à ce climat d'instabilité, une voie incontournable s'impose : s'engager dans une démarche environnementale en mettant en place un Système de Management Environnemental (SME).

La mise en place progressive de réglementation selon le SME vise par exemple à normer la consommation de ressources ce qui a conduit les entreprises à adopter des stratégies variées de réactives à pro actives (Sharma, 2000). En effet, le SME insiste sur le fait qu'il faut passer d'une logique palliative à une démarche préventive, réduisant ainsi la pollution à la source par l'intégration des préoccupations environnementales dans la gestion quotidienne de l'entreprise. En effet, une anticipation à la réglementation environnementale permet à l'entreprise d'avoir plus de temps de se conformer à la législation qu'une adaptation. Ceci est de nature à diminuer les perturbations dans les processus de production, ce qui permet à l'entreprise de se procurer un avantage compétitif par rapport à ses concurrents. De plus, ces efforts sont considérés comme réactives dont le but est d'éviter les sanctions.

La certification ISO I400 I ne fixe pas de limite ou d'objectifs au niveau de l'impact environnemental, mais elle a surtout vocation à garantir sur les marchés mondiaux une certaine qualité de management environnemental auprès d'un large public. A ce titre, Boiral (1998) remarque que la gestion de la qualité (norme ISO 9000) et la gestion environnementale (norme ISO |400I) procèdent d'une même recherche de citoyenneté de la part des entreprises. Ainsi, les référentiels ISO 9000 ou ISO |400| véhiculent une image de rigueur, d'objectivité, de précision et de contrôle empruntés aux sciences exactes (Boiral, 2006). Dès lors, il faudrait commencer par une analyse environnementale. Le règlement Européen du système de Management Environnemental (EMAS) définit l'analyse environnementale comme « une analyse préliminaire approfondie des problèmes, de l'impact et des résultats en matières d'environnement liés aux activités menées sur un site ». 
Cette démarche d'analyse nous donne une image de l'entreprise à un moment donné pour avoir plus d'informations sur les défaillances, les conséquences environnementales ainsi que les actions correctrices.

Dans ce contexte, la modélisation fonctionnelle et dysfonctionnelle du système de production facilite la certification aux normes (ISO) en permettant de documenter l'entreprise tout en capitalisant les connaissances et par voie de conséquences la maîtrise des AES. Ainsi et pour ce qu'est qui est de la modélisation fonctionnelle, notre choix s'est porté sur la méthode GRAICO qu'est qui est complétée, pour ce qu'est qui est de la modélisation dysfonctionnelle, par la méthode Analyse des Défaillances et leurs Conséquences (ADC).

\section{Méthodes de modélisation}

\section{I - Modélisation de l'entreprise}

La modélisation d'entreprise (Vernadat, 1999) s'impose peu à peu comme un outil indispensable pour capitaliser la connaissance de l'entreprise et l'appréhender sous ses différents aspects (structurels, fonctionnel, comportemental, informationnel, organisationnel ou autre). Cette compréhension de l'existant permet de le contrôler, de le simuler, de l'analyser ou même de le réorganiser afin d'en améliorer les performances (Scheer, 1999).

Selon Le Moigne (1999) la modélisation d'entreprise est « l'action d'élaboration et de construction intentionnelle par composition de symboles, de modèles susceptibles de rendre tangible un phénomène perçu complexe ». Conséquemment, la modélisation facilite la certification aux normes ISO 9000 et ISO |400| en permettant de documenter l'entreprise tout en capitalisant les connaissances.

\section{2- Les méthodes de modélisation}

La situation actuelle des méthodes de modélisation se résume en (Doumeingts, 1984 ; Fenie, 1994 ; Glassey, 2002) : méthodes et architectures de référence (c'est l'exemple de CIMOSA et PERA) et méthodes opérationnelles (comme : SADT, MERISE, UML, OSSAD, OLYMPIOS, GRAI et GRAICO).

La tendance actuelle est de modéliser l'entreprise par ses processus. En effet, cette modélisation est devenue une pratique courante depuis que les travaux normatifs ont contribué à généraliser cette approche notamment dans le domaine de la qualité (Glassey, 2002). C'est ainsi que la norme ISO 9000/2000 a défini quatre types de processus générique : le processus de management (de pilotage ou de direction), le processus de réalisation (opérationnel), le processus support (processus de soutient ou processus ressources) et le processus de mesure. II en est de même pour la norme ISO |400| puisque celle-ci transpose à l'environnement les règles de management de la qualité. A cet effet, la méthode GRAICO permet la modélisation des processus de production de l'entreprise et donne ainsi une image du système de production par le formalisme GRAICO.
De ce fait, la méthode GRAICO répond au besoin de modélisation par processus dont l'objectif est la mise en place de I'ISO | 4001 .

Conséquemment, la méthode GRAICO permet l'analyse du fonctionnement de l'entreprise par le biais des réseaux GRAICO qui permettent donc la modélisation par processus (processus de réalisation et processus supports) dont le but est de déterminer et d'évaluer les aspects environnementaux qu'il faudra maitriser par la méthode ADC. D'où le couplage GRAICO/ADC, objet de cette étude.

\section{3- Présentation succincte des méthodes retenues : GRAI CO-ADC} Suite à de nombreuses applications, la méthode GRAl a évolué, en particulier, avec l'introduction du sous système d'exploitation (Fenie 94 ; Marcotte, 1995). Cette évolution est due à l'émergence d'un fonctionnement sur événement au niveau du système décisionnel, en complément au fonctionnement périodique à des horizons de décision plus élevés (détectés à l'origine du modèle GRAI). En effet, plus on est proche du système physique de production plus, les contraintes que rencontre le pilotage sont imposées par les événements qui se produisent dans ce système. A ce niveau, il s'agit d'assurer la synchronisation qui met en jeu un ensemble d'éléments liés à l'exploitation du système physique : les moyens, les outils, les stocks, la qualité, l'environnement, la maintenance...

La gestion des modes dégradés, dans un système de production, joue un rôle primordial (Marcotte, 1995).

En effet, la supervision du procédé physique, sa gestion lorsqu'il fonctionne de façon non optimale, la gestion technique, ...., sont des fonctions de conduites importantes, qui sont rarement prises en compte. La méthode GRAICO est une méthode qui permet la modélisation des procédés physiques par émergence du système d'exploitation. Le système de conduite d'un système de production, tel qu'on a pu l'appréhender jusqu'alors, s'étend aux niveaux les plus élevés de la structure de la gestion de l'entreprise, et ce dans les détails de la commande des procédés physiques de fabrication. Les fonctions suscitées se rencontrent dans la plus part des procédés industriels.

Afin d'intégrer ces fonctions que l'on qualifiera d'exploitation, à la fois au niveau structure et au niveau intermédiaire de conduite, il a été nécessaire d'introduire un niveau intermédiaire de conduite appelé « système d'exploitation ». Elle s'effectue suivant cinq étapes (Vallespir, 1987) : initialisation, analyse du procédé, structuration du système d'exploitation, description détaillée des fonctions d'exploitation et détermination détaillée de la structure des données.

La modélisation est basée sur une décomposition hiérarchique du procédé. De ce fait, un procédé sera envisagé comme une hiérarchie d'activités visant à assurer des objectifs d'exploitation illustré dans la figure I. Ce modèle, listant les activités utilisées, mais aussi les liens qui existent entre elles et leur nature, sera appelé Structure Fonctionnelle de Procédé (SFP). 
La finalité de l'analyse environnementale étant l'identification des AES. L'analyse des Défaillances et des Conséquences (ADC) illustrée dans le tableau I vient compléter la méthode GRAICO afin de pouvoir maîtriser les défaillances pouvant être à l'origine de ces impacts. En effet, l'ADC a pour but de fournir une base de connaissance au moyen d'un tableau guidant l'analyse, de la description du mode de défaillance d'un composant matériel à celle de ses conséquences et des actions correctrices à entreprendre. Cette étude des défaillances sera naturellement basée sur l'exploitation des modes définis précédemment. Les informations principales sont listées dans un tableau.

\section{3- Application}

Le but de l'usage combiné des méthodes GRAICO/ADC est de modéliser, dans un premier temps, les activités d'une entreprise notamment le système physique par la méthode GRAICO, ce formalisme nous donne une image de l'usine ce qui nous facilite la compréhension du système de production correspondant. L'ADC vient compléter ce travail par une formalisation dysfonctionnelle de ce système. Afin d'illustrer nos propos, nous nous sommes intéressés à une cimenterie algérienne dont le processus de production est rappelé ci-après.

\section{I- Rappel succinct du processus de fabrication du ciment}

De la carrière on récupère de l'argile et du calcaire où ils subissent un premier broyage ensuite ils sont acheminés vers les silos d'homogénéisation ils arrivent sous forme de tas. Suite à un ajout de pozolane (minimise le prix de revient) et de minerai de fer (diminue la température), on effectue un pesage (LSF), les grains devront passer un tamis de 90 Im. Le mélange va encore subir un broyage à cru puis la farine déversée dans les cyclones subit un pré échauffement puis échauffements dans les fours. Le clinker obtenu est mis en silos de stockage pour subir un dosage en gypse (augmente le temps de reprise) et enfin un dernier broyage. On parle dès lors de ciment.

\section{2- Modélisation du processus de fabrication par les réseaux GRAICO}

Cette phase a pour objectif de modéliser toutes les activités de l'entreprise et de déterminer les liens entre ses activités. C'est un état des lieux de la cimenterie qui nous facilitera la tâche pour l'analyse environnementale.

La figure I représente un extrait des réseaux GRAICO de la cimenterie d'Ain-Touta (Boubaker, 1997).

L'exploitation des réseaux GRAICO nous permet de mener une analyse environnementale de ladite cimenterie (tableau I).

Dans la figure I, nous avons utilisé des outils de formalisation spécifique à la méthode GRAICO dont les significations sont rappelées dans le tableau 2. La hiérarchisation des AES se fait selon les critères de pondération représentés dans le tableau 3. Le produit de ces trois critères, nous permet de déterminer l'Aspect Environnemental Significatif (AES). Notons que nous avons retenu en accord avec le responsable environnement de la cimenterie étudiée qu'un AES est significatif si ce dernier dépasse la valeur 12 ce qui est le cas pour l'activité de cuisson (activité $n^{\circ 5}$ ). Pour maitriser cette activité, dont l'aspect environnemental est significatif, nous poursuivons l'analyse par l'élaboration de I'ADC.

Tableau I : Extrait de l'analyse Environnementale de la cimenterie d'Ain-Touta, Algérie.

\begin{tabular}{|c|c|c|c|c|c|c|c|c|}
\hline \multirow{2}{*}{$\begin{array}{c}N^{\circ} \\
\text { Activité }\end{array}$} & \multirow[t]{2}{*}{ Activité } & \multirow[t]{2}{*}{ Aspect } & \multirow[t]{2}{*}{ Impact } & \multicolumn{3}{|c|}{ Cotation } & \multirow[t]{2}{*}{$N^{\circ} A E S$} & \multirow[t]{2}{*}{$A E S$} \\
\hline & & & & $F$ & $M$ & $N$ & & \\
\hline \multirow[t]{2}{*}{2} & \multirow{2}{*}{$\begin{array}{l}\text { Transport et } \\
\text { stockage farine } \\
\text { crue }\end{array}$} & $\begin{array}{l}\text { Emission de } \\
\text { poussières }\end{array}$ & $\begin{array}{l}\text { Pollution } \\
\text { atmosphérique }\end{array}$ & 3 & 4 & 4 & 201 & 11 \\
\hline & & $\begin{array}{l}\text { Déversement de } \\
\text { matières }\end{array}$ & $\begin{array}{l}\text { Pollution du sol et } \\
\text { altération du site }\end{array}$ & 3 & 4 & 4 & 202 & 11 \\
\hline 3 & $\begin{array}{l}\text { Préparation } \\
\text { matières premières }\end{array}$ & $\begin{array}{l}\text { Emission de } \\
\text { poussières }\end{array}$ & $\begin{array}{l}\text { Pollution } \\
\text { atmosphérique }\end{array}$ & 3 & 4 & 1 & 303 & 8 \\
\hline \multirow[t]{2}{*}{4} & \multirow[t]{2}{*}{$\begin{array}{l}\text { Alimentation } \\
\text { farine crue }\end{array}$} & $\begin{array}{l}\text { Émission de } \\
\text { poussières }\end{array}$ & $\begin{array}{l}\text { Pollution } \\
\text { atmosphérique }\end{array}$ & 3 & 4 & 1 & 404 & 8 \\
\hline & & $\begin{array}{l}\text { Déversement de } \\
\text { la farine }\end{array}$ & Pollution du sol & 3 & 4 & 1 & 405 & 8 \\
\hline \multirow[t]{2}{*}{5} & \multirow[t]{2}{*}{ Cuisson } & $\begin{array}{l}\text { Émission de } \\
\text { poussières }\end{array}$ & $\begin{array}{l}\text { Pollution du sol et } \\
\text { altération du site }\end{array}$ & 4 & 4 & 4 & 506 & 12 \\
\hline & & $\begin{array}{l}\text { Consommation } \\
\text { d'énergie }\end{array}$ & $\begin{array}{l}\text { Appauvriss ement } \\
\text { ressources naturelles }\end{array}$ & 4 & 4 & 4 & 507 & 12 \\
\hline 6 & $\begin{array}{l}\text { Filtration atelier } \\
\text { cuisson }\end{array}$ & $\begin{array}{l}\text { Émission de } \\
\text { poussières }\end{array}$ & $\begin{array}{l}\text { Pollution } \\
\text { atmosphérique, }\end{array}$ & 2 & 4 & 4 & 607 & 10 \\
\hline 7 & $\begin{array}{l}\text { Transport et } \\
\text { stockage clinker }\end{array}$ & $\begin{array}{l}\text { Émission de } \\
\text { poussières }\end{array}$ & Pollution du sol & 2 & 2 & 4 & 708 & 8 \\
\hline
\end{tabular}


Figure I : Extrait des érseaux GRAICO de la cimenterie Ain-touta, Algérie

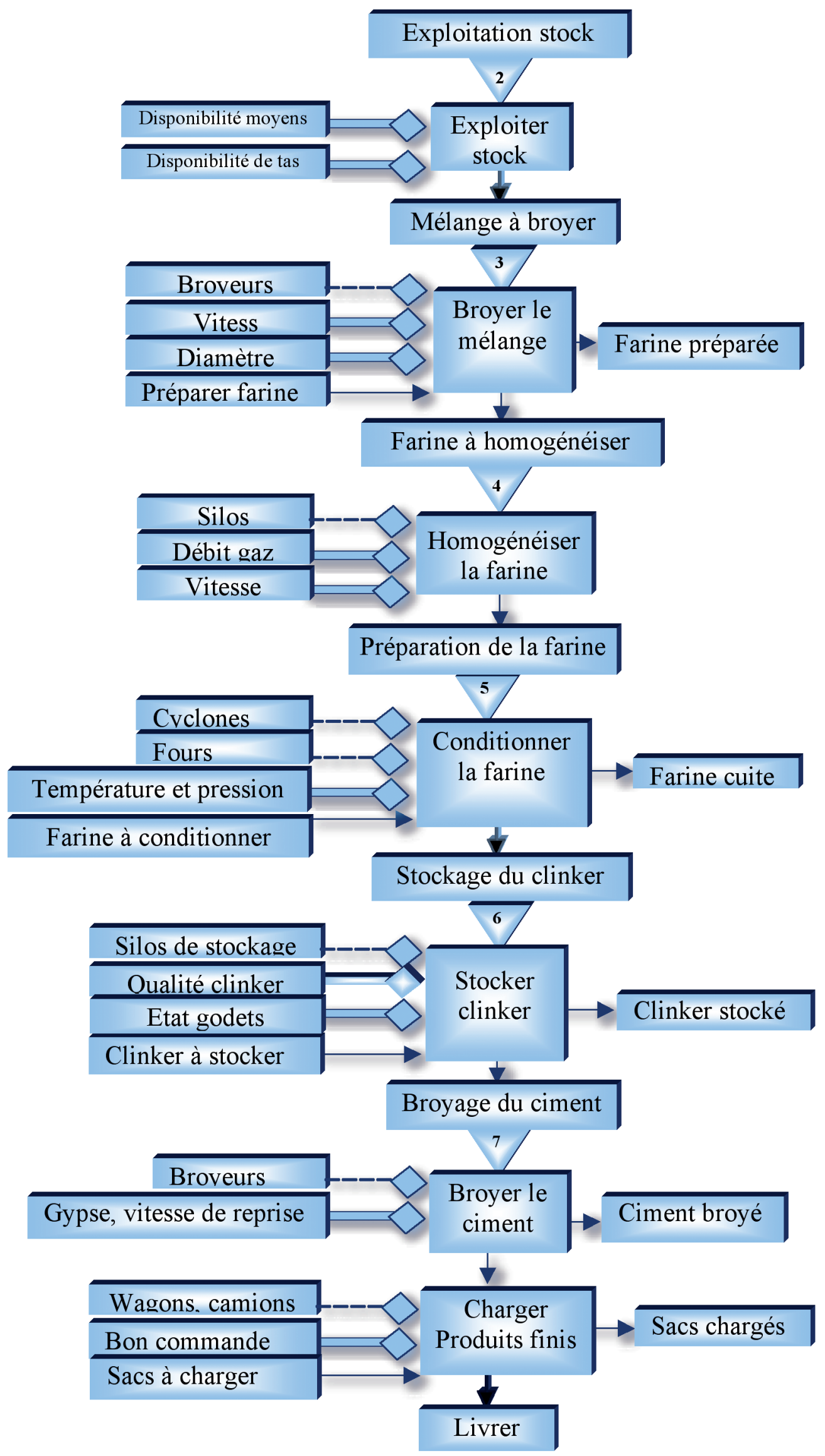


Tableau 2 : Outils de formalisme GRAICO

\begin{tabular}{|c|c|c|}
\hline Flux & Flux traités & $\begin{array}{c}\text { Flux su pports à } \\
\text { l'activité }\end{array}$ \\
\hline Matières & $\longrightarrow$ & \\
\hline Energie & $\longrightarrow$ & - \\
\hline Informations & $\longrightarrow$ & \\
\hline Evénements & $\longrightarrow$ & \\
\hline
\end{tabular}

Tableau 3 : Critères de pondération pour la détermination des AES.

\begin{tabular}{|c|c|c|c|c|c|c|c|}
\hline \multirow{2}{*}{$\begin{array}{l}\text { Critères de } \\
\text { pondération }\end{array}$} & \multirow[t]{2}{*}{ Appellation } & \multicolumn{6}{|c|}{ Cotation } \\
\hline & & Indice 1 & Note & Indice 2 & Note & Indice 3 & Note \\
\hline $\begin{array}{l}\text { Fréquence } \\
\text { d'apparition }\end{array}$ & $\mathrm{F}$ & Une fois/an & 1 & $\begin{array}{l}\text { Une } \\
\text { fois/mois }\end{array}$ & 2 & Continue & 4 \\
\hline $\begin{array}{l}\text { Niveau de } \\
\text { maitrise }\end{array}$ & $\mathrm{M}$ & $\begin{array}{l}\text { Détection } \\
\text { systématique }\end{array}$ & 1 & $\begin{array}{l}\text { Détection } \\
\text { rapide }\end{array}$ & 2 & $\begin{array}{l}\text { Non } \\
\text { détection }\end{array}$ & 4 \\
\hline $\begin{array}{l}\text { Nuisance de } \\
\text { l'aspect }\end{array}$ & $\mathrm{N}$ & Local & 1 & Usine & 2 & Régional & 4 \\
\hline
\end{tabular}

\section{3- Formalisation dysfonctionnelle par ADC}

D'habitude cette méthode est utilisée à des fins de production l'idée qu'on a eu c'est de l'utiliser à des fins environnementales suite à l'analyse environnementale effectuée pour toutes activités du système de production de la cimenterie étudiée (tableau I). Notons que GRAICO nous a aidé à avoir une vue sur cette cimenterie.

Signalons que dans le tableau ci-après et pour ce qu'est des conséquences environnementales, nous proposons dans la colonne cotation ce qui suit:

$$
\begin{aligned}
& \text { Plainte des riverains }=\left\{\begin{array}{l}
1 \text { si une } \frac{\text { fois }}{\text { an }} \\
4 \text { si une } \frac{\text { fois }}{\text { mois }}
\end{array}\right. \\
& \text { Capacité de détection }=\left\{\begin{array}{l}
1 \text { systématiqu } \\
4 \text { inéxistante }
\end{array}\right.
\end{aligned}
$$

L'examen des résultats obtenus montre que certes l'AES est tolérable, mais toutefois il est souhaitable de le rendre acceptable par l'adoption d'une stratégie environnementale proactive. De ce fait, les actions environnementales peuvent se matérialiser par (Boubaker et al., 20I I) :
- un recours aux combustibles et matière premières de substitution permettant de réduire la consommation de ressources naturelles telles que la calcaire et la marne, et d'améliorer le bilan $\mathrm{CO}_{2}$,

- la substitution des électro filtres par des filtres à manches à efficacité énergétique accrue ; car leur mise en place permet de supprimer les tours de conditionnement pour les broyeurs Cette suppression permet une énorme économie d'eau (économie de $40 \mathrm{~m}^{3}$ d'eau/h soit $200.000 \mathrm{~m}^{3} / \mathrm{an}$ ).

- une maintenance pro active des cyclones.

\section{Conclusion}

Pour pouvoir mettre en place l'ISO |400 I, nous avons eu besoin dans un premier temps de modéliser la cimenterie par le formalisme GRAICO ce qui nous a permis de faire un état des lieux sur le processus de fabrication de celleci. Dans un second temps, nous avons procédé à l'analyse environnementale, tâche qui sans ce formalisme aurait pris plus de temps, nous avons finis par une mise à plat des connaissances des effets sur l'environnement des processus de production.

La mise en place ISO I400 I est l'occasion de déployer, en interne, une politique d'intégration « à la source » des exigences environnementales. Dans ce contexte, nous avons constaté lors de notre passage à la cimenterie que les employés de la cimenterie ne sont pas suffisamment informés sur les questions de l'environnement. 
Tableau 4 : Analyse des défaillances et de leurs conséquences au sein de la cimenterie étudiée.

\begin{tabular}{|c|c|c|c|c|c|}
\hline $\begin{array}{c}N^{\circ} \\
\text { Activité }\end{array}$ & Matériel & Défaut matériel & $\begin{array}{c}\text { Défaillance } \\
\text { fonctionnelle }\end{array}$ & $\begin{array}{l}\text { Activité } \\
\text { affectée }\end{array}$ & $\begin{array}{c}\text { Situation } \\
\text { initiale }\end{array}$ \\
\hline & $\begin{array}{l}\text { Buses (Tour de } \\
\text { conditionnement) }\end{array}$ & $\begin{array}{l}\text { Défaut de Buse (injection } \\
\text { d'eau liquide non vapeur) }\end{array}$ & $\begin{array}{l}\text { Perte pot/ } \\
\text { marche }=1\end{array}$ & $\begin{array}{l}\text { Filtration des } \\
\text { poussières }\end{array}$ & $\begin{array}{l}\text { Marche pot/ } \\
\text { arrêt }=1\end{array}$ \\
\hline & $\begin{array}{l}\text { Vice sans fin (Tour } \\
\text { de conditionnement) }\end{array}$ & Colmatage de la vice sans fin & $\begin{array}{l}\text { Perte pot/ } \\
\text { marche }=1\end{array}$ & $\begin{array}{l}\text { Filtration des } \\
\text { poussières }\end{array}$ & $\begin{array}{l}\text { Marche pot/ } \\
\text { arrêt }=1\end{array}$ \\
\hline 5 & $\begin{array}{l}\text { Redresseurs des } \\
\text { électro-filtres (Tour } \\
\text { de conditionnement) }\end{array}$ & $\begin{array}{l}\text { 2/4 redresse urs captent la } \\
\text { poussi ère (r ejet de poussières } \\
\text { dans l'air) }\end{array}$ & $\begin{array}{l}\text { Perte pot/ } \\
\text { marche }=1\end{array}$ & $\begin{array}{l}\text { Filtration des } \\
\text { poussières }\end{array}$ & $\begin{array}{l}\text { Marche pot/ } \\
\text { arrêt }=1\end{array}$ \\
\hline
\end{tabular}

\section{Conséquences environn ementales}

\begin{tabular}{|c|c|c|c|c|c|}
\hline \multirow[t]{2}{*}{$N^{\circ} A E S$} & \multirow[t]{2}{*}{ Nature } & \multirow[t]{2}{*}{ Durée de pollution } & \multicolumn{3}{|c|}{ Cotation } \\
\hline & & & $\begin{array}{l}\text { Plainte des } \\
\text { riverains }\end{array}$ & $\begin{array}{l}\text { Capacité de } \\
\text { détection }\end{array}$ & $\begin{array}{l}\text { Degré des } \\
\text { conséquences }\end{array}$ \\
\hline & $\begin{array}{l}\text { Retombée des poussières sur } \\
\text { le sol. } \\
\text { Émission des pouss ières }\end{array}$ & $\begin{array}{l}1 \text { heure } \\
\text { (le temps de stabiliser le four) }\end{array}$ & 1 & 1 & Faible \\
\hline 506 & $\begin{array}{l}\text { Retombée des poussières sur } \\
\text { le sol }\end{array}$ & $\begin{array}{l}\text { 1heure } \\
\text { (le temps de stabiliser le four) }\end{array}$ & 1 & 1 & Faible \\
\hline
\end{tabular}

\section{Actions cor rectrices}

\begin{tabular}{|c|c|c|c|c|c|}
\hline $\begin{array}{c}\text { Actions } \\
\text { (options } \\
\text { techniques) }\end{array}$ & \multicolumn{2}{|c|}{ Délai de la correction } & $\begin{array}{l}\text { Degré de } \\
\text { maîtrise }\end{array}$ & $\begin{array}{l}\text { Communication } \\
\text { internelexterne }\end{array}$ & \\
\hline Élément contrôlé & Réduire AES506 & $\begin{array}{l}\text { Supprimer AES506 } \\
\text { (mod ifi cation } \\
\text { technologiq ue) }\end{array}$ & & & \\
\hline $\begin{array}{l}\text { Replacer les } \\
\text { buses }\end{array}$ & $\begin{array}{l}\text { Maintenance des } \\
\text { filtres }\end{array}$ & $\begin{array}{l}\text { Placer des filtres à } \\
\text { manches }\end{array}$ & 1heure & $\begin{array}{l}\text { Fort degré de } \\
\text { maîtrise }\end{array}$ & Direction de l'environnement \\
\hline Dé colmatage & $\begin{array}{l}\text { Maintenance des } \\
\text { cyclones }\end{array}$ & $\begin{array}{l}\text { Maintenance des } \\
\text { cyclones }\end{array}$ & 1heure & $\begin{array}{l}\text { Fort degré de } \\
\text { maîtrise }\end{array}$ & $\begin{array}{l}\text { Direction de l'environnement } \\
\text { Direction de l'environnement }\end{array}$ \\
\hline
\end{tabular}


Un autre constat concerne l'existence de la division du travail et des compétences, il apparaît nécessaire de faire un décloisonnement entre les différentes fonctions de l'entreprise (fonction de l'environnement, de production et de méthodes). De ce fait, la construction de l'ISO I400| n'est pas seulement descendante, elle est surtout transversale.

La mise en place de I'ISO |400I, ne se réduit pas à des mesures techniques, elle suppose une responsabilité beaucoup plus large, plus grande, c'est d'abord une culture et un état d'esprit. C'est pourquoi, nous préférons le terme de construction de l'ISO |400| plutôt que de mis en place.

\section{Références bibliographiques}

Boiral, O., ( | 998), « ISO | 400 I: Against the tide of modern management? » Journal of General Management, Vol. 24, Nº I, pp. 35-52.

Boiral, O., (2006), « La certification ISO I 400 I: une perspective néo institutionnelle», Management International, Vol. 10, No. 3, p. 67-79.

Boubaker, L., (1997), Mise en place de l'approche CIM dans un système de gestion de production : cas d'une entreprise algérienne. Mémoire de Magister soutenu à l'Université de Batna, Algérie.

Boubaker, L., Djebabra, M., Chaabane, H., and Leal Filho , W., (20 I I), « Environmental impacts typology: a methodological proposal». International Journal of Sustainable Development, Vol. I4, NI/2,20I I, pp. I22-140.

Doumeingts, G., (1984). La méthode GRAl : méthode de conception des systèmes en productique. Thèse de Doctorat soutenue à l'Université Bordeaux-I, France.

Fenie, P., (1994). GRAICO: Méthode de modélisation et de conception des systèmes d'exploitation de systèmes de production. Thèse de Doctorat soutenue à l'Université Bordeaux-I, France.

Glassey, O. et Chappelet, J.-L., (2002), « Comparaison de trois techniques de modélisation de processus: ADONIS, OSSAD et UML », Working paper, Lausanne : IDHEAP.

Le Moigne, J.L., (1999). La modélisation des systèmes complexes. Dunod, Paris, $2^{\mathrm{ème}}$ édition.

Marcotte, F., (1995). Contribution à la modélisation des systèmes de production : extension du modèle GRAI. Thèse de Doctorat soutenue à I'Université Bordeaux-I, France.

Mirdamadi S., (2009). Modélisation du processus de pilotage d'un atelier en temps réel à l'aide de la simulation en ligne couplée à l'exécution. Thèse de doctorat soutenue à I'Institut National Polytechnique de Toulouse, France.
Sharma, S., (2000), « Managerial interpretations and organizational context as predictors of corporate choice of environmental strategy » Academy of Management Journal, Vol.43, No 4, pp.68I-697

Scheer, W.A., (1999). ARIS - Business Process Modeling. Springer-Verlag, Berlin.

Vernadat, F., (1999). "Techniques de Modélisation en Entreprise : Applications aux Processus Opérationnels », Editions Economica. 